\title{
Association of fetuin-A with incident type 2 diabetes: results from the MONICA/KORA Augsburg study and a systematic meta-analysis
}

\author{
Chaterina Sujana ${ }^{1,2}$, Cornelia Huth ${ }^{2,3}$, Astrid Zierer2, Sophie Meesters², Julie Sudduth-Klinger ${ }^{4,5}$, \\ Wolfgang Koenig ${ }^{6,7,8}$, Christian Herder ${ }^{3,9}$, Annette Peters ${ }^{2,3,7}$ and Barbara Thorand ${ }^{2,3}$
}

${ }^{1}$ Institute for Medical Informatics, Biometry and Epidemiology, Ludwig-Maximilians Universität, Munich, Germany, ${ }^{2}$ Institute of Epidemiology II, Helmholtz Zentrum München, German Research Center for Environmental Health, Neuherberg, Germany, ${ }^{3}$ German Center for Diabetes Research (DZD), Munich-Neuherberg, Germany, ${ }^{4}$ Tethys Bioscience, Emeryville, California, USA, ${ }^{5} \mathrm{HDF}$ Comprehensive Cancer Center, University of California San Francisco, San Francisco, California, USA, ${ }^{6}$ Deutsches Herzzentrum München, Technische Universität München, Munich, Germany, ${ }^{7}$ German Centre for Cardiovascular Research (DZHK), Partner Site Munich Heart Alliance, Munich, Germany, ${ }^{8}$ Department of Internal Medicine II-Cardiology, University of Ulm Medical Center, Ulm, Germany, and ${ }^{9}$ Institute for Clinical Diabetology, German Diabetes Center, Leibniz Center for Diabetes Research at Heinrich Heine University Düsseldorf, Düsseldorf, Germany

Correspondence should be addressed to $B$ Thorand

Email

thorand@helmholtzmuenchen.de

\begin{abstract}
Objective: We investigated the association of circulating fetuin-A with incident T2D particularly examining potential sex differences. Additionally, we determined whether putative associations were independent of subclinical inflammation, adiponectin and liver fat content.

Design: Case-cohort study plus systematic meta-analysis.

Methods: We investigated the association between baseline fetuin-A levels and incident T2D in the MONICA/KORA Augsburg study using Cox proportional hazards analyses. Furthermore, we conducted a systematic review within PubMed and EMBASE and pooled association estimates of eligible studies with the MONICA/KORA Augsburg data using a DerSimonian-Laird random effects model.

Results: Within MONICA/KORA Augsburg, 930 participants developed incident T2D (median follow-up: 14 years). We observed a significant association between fetuin-A and T2D risk after multivariable adjustment including C-reactive protein and adiponectin. The strength of the association was similar in males and females ( $P$ value for sex interaction $>0.55)$. Seven eligible published studies were identified in addition to the MONICA/KORA Augsburg study for the meta-analysis. The pooled hazard ratio $(95 \% \mathrm{Cl})$ for incident T2D per 1 standard deviation (s.D.) increment of fetuin-A was 1.24 (1.14-1.34) for the multivariable adjusted model. Our sex-stratified meta-analysis yielded relative risk estimates per 1 S.D. of 1.19 (1.04-1.38) in males and 1.29 (1.15-1.46) in females. Further individual adjustment for subclinical inflammation, adiponectin and liver fat content had almost no impact on the strength of the association. Conclusions: Higher fetuin-A levels are associated with incident T2D in both males and females independently of subclinical inflammation, adiponectin and liver fat content.

European Journal of Endocrinology (2018) 178, 389-398
\end{abstract}

\section{Introduction}

The number of people with diabetes is predicted to increase by nearly $60 \%$ from around 400 million in 2015 to over 640 million in 2040 (1). Diabetes is projected to become one of the leading causes of death, and type 2 diabetes (T2D) is the most frequent type of

www.eje-online.org

https://doi.org/10.1530/EJE-17-1053 (c) 2018 European Society of Endocrinology Printed in Great Britain diabetes (2). There are several different mechanisms underlying the pathogenesis of T2D. Understanding its complex pathophysiology is important for management of the disease and the development of prevention strategies (3). 
Human fetuin-A or alpha-2 Heremans Schmid glycoprotein is an endogenous glycoprotein secreted by the liver that plays a role in insulin resistance in $\mathrm{T} 2 \mathrm{D}$ patients (4) and that is suggested to be a novel risk marker for T2D in non-diabetic populations $(5,6,7)$. However, some studies reported significant associations in females only $(8,9)$. Furthermore, previous studies used varying degrees of adjustment, and it is not entirely clear whether observed associations are mediated or confounded by subclinical inflammation $(5,8,10)$, adiponectin $(10,11)$ or liver fat content $(5,8,9,11)$.

The present study aimed to further investigate the association between fetuin-A and incident T2D, particularly examining potential sex differences and addressing the impact of subclinical inflammation, adiponectin and liver fat content on the observed associations. As a first step, we conducted a case-cohort study using unpublished data from the Monitoring of Trends and Determinants in Cardiovascular Diseases (MONICA) Augsburg/Cooperative Health Research in the Region of Augsburg (KORA) study. As a second step, to assess the association between circulating fetuin-A and risk of T2D with the maximum of statistical power, we performed a systematic review and pooled data from MONICA/KORA Augsburg together with all eligible published studies.

\section{Subjects and methods}

\section{The MONICA/KORA Augsburg case-cohort study}

\section{Study population}

A prospective case-cohort study was conducted within the MONICA/KORA Augsburg study population including three independent cross-sectional population-based surveys, S1-S3, conducted between 1984 and 1995. The MONICA/KORA Augsburg study was designed to estimate the prevalence and distribution of cardiovascular risk factors among 13427 men and women aged 25-74 years in the region of Augsburg, Southern Germany. The study design has been previously described in detail $(12,13)$. Informed consent was provided by all study participants, and the ethics committee of the Bavarian Chamber of Physicians approved the study protocol. The study complies with the principles outlined in the Declaration of Helsinki.

In the present MONICA/KORA Augsburg case-cohort study, we restricted the source population to those aged $35-74$ years ( $n=10718$ persons) due to the low incidence of T2D under the age of 35 years. The follow-up period was restricted until 2009. After exclusion of 1187 participants without blood sample, 1 newly identified duplicate, 508 participants with prevalent T2D, 25 persons who developed other types of diabetes, 473 persons without follow-up information for T2D and 55 persons with an observation time $\leq 365$ days, the final source population comprised 8469 participants. Out of these, a random subcohort of 1991 persons was selected stratified by sex and survey. A total of 930 incident T2D cases (43.4\% were females) were included; 217 of these cases stemmed from the sub-cohort. The study participants were followed for a median of 14.3 years (25th and 75 th percentiles were 11.2 and 19.2 years, respectively).

\section{Assessment of T2D risk factors}

Sociodemographic, clinical and lifestyle parameters were assessed as previously described in detail (14). Serum and plasma samples stored at $-80^{\circ} \mathrm{C}$ were used to analyse biomarkers $(15,16)$.

\section{Fetuin-A measurement}

Fetuin-A was measured in serum using ultrasensitive molecular counting technology (MCT; Singulex, Alameda, California, USA) (17). The fetuin-A assay used a recombinant fetuin-A protein (1184-P1-050) from R\&D Systems, a mouse monoclonal anti-human fetuin-A capture antibody (MAB1184) from R\&D Systems and a polyclonal goat anti-fetuin-A detection antibody (AF1184; R\&D Systems). The intra- and inter-assay coefficients of variation were $10 \%$ and $11 \%$, respectively.

\section{Assessment of incident type 2 diabetes}

As described previously (13), incidence of T2D was initially assessed from patients' self-report of physician-diagnosed diabetes and the use of antidiabetic medication through written follow-up questionnaires sent to all MONICA/ KORA S1-S3 study participants in 1997/1998, in 2002/2003 and in 2008/2009. Additionally, all participants from the first survey were invited to participate in a follow-up examination conducted in 1987/1988. All self-reports of physician-diagnosed diabetes were validated by contacting the treating physician or medical chart review and type of diabetes, and the year of diagnosis were assessed.

\section{Statistical analysis}

Cox proportional hazards models modified for the casecohort study design using Barlow's method (18) were used 
to evaluate the association between fetuin- $\mathrm{A}$ and incident T2D. Results are given as hazard ratios (HRs) with 95\% confidence intervals (CIs) per sex-specific quartile of fetuin-A. We also evaluated HRs per 1 standard deviation (S.D.) increment of fetuin-A, considering natural logtransformed fetuin-A as a continuous variable. Missing information on fetuin-A and other covariates were less than $22 \%$ and were imputed using 20-fold multiple imputation by chained equations (MICE) (19), performed using $\mathrm{R}$, version 3.2.3 (20) and R package mice, version 2.25 (21). Additional variation due to imputation was taken into account according to the Rubin's rules for multiple imputation (22).

Cox proportional hazards models were initially adjusted for age, sex and the three surveys (model 1). Then, we further adjusted for classical T2D risk factors by adding body mass index, smoking status, alcohol intake, physical activity, high-density lipoprotein level, actual hypertension and parental history of diabetes (model 2). We further adjusted for daily consumption of coffee, meat and whole grain products (model 3 ). In addition, CRP, as a marker of subclinical inflammation and adiponectin were separately added to the Cox models (all natural logtransformed) to assess their mediating or confounding effect on the association of fetuin-A and incident T2D (models 4 and 5). In addition to analyses in the total study sample, we examined sex-specific associations and formally tested for sex differences by inclusion of an interaction term between sex and level of fetuin-A.

Apart from multiple imputations, all statistical analyses were carried out with the statistical software SAS 9.3 edition (SAS Institute, Cary, North Carolina, USA). As far as nothing else is mentioned, we applied a significance level of 0.05 to all analyses.

\section{Systematic meta-analysis}

The systematic literature search strategy to retrieve all published eligible studies is reported in the Supplementary Text 1 (see section on supplementary data given at the end of this article).

We pooled effect estimates with 95\% CIs from the eligible studies using the DerSimonian and Laird random effects model assuming true heterogeneity between the reported individual study associations $(23,24)$. Inversevariance fixed effects models (25) were calculated for comparison. A significance level of 0.05 was applied. Reported HRs and odd ratios for incident T2D were assumed to approximate relative risks (RRs). We calculated pooled RR estimates adjusted for as many established risk factors as possible and for age and sex plus minimum number of other covariates only. If only one adjustment model was reported, the reported association was included in the minimum and in the multivariable adjusted model.

To investigate whether there were sex differences in the association between fetuin-A and risk of T2D, studies that reported sex-specific associations were pooled in a subgroup analysis. Further subgroup analyses were carried out by calculating pooled estimates defined by age ( $<65$ years; $\geq 65$ years), ethnicity (mixed; predominantly Caucasian), study size ( $<100$ incident cases; $\geq 100$ incident cases) and study quality using a quality score based on an adapted version of the Newcastle-Ottawa Scale (NOS) for cohort studies (26) (Supplementary Text 2) (i.e. risk of bias: NOS <median score; NOS $\geq$ median score). A sensitivity analysis was performed, excluding studies conducted in specific high-risk populations (such as prediabetic populations).

To establish whether the association between fetuin-A and T2D is independent of subclinical inflammation (assessed by CRP, interleukin-6 (IL6) and tumor necrosis factor (TNF)alpha), adiponectin levels and liver fat content (assessed either directly through computed tomography (CT) imaging or indirectly through measurement of liver enzymes), we performed additional analyses including only studies that presented results with adjustment for these factors.

We carried out statistical tests for heterogeneity between study-specific association estimates using chisquared Cochran's $Q$ test for heterogeneity (27), applying a significance level of 0.10 . The test examines the null hypothesis that included studies evaluate a common effect (28). We quantified the heterogeneity with the I-squared statistic, which describes the proportion of total variation between individual study estimates due to heterogeneity rather than due to chance or sampling error (29). The value of I-squared statistic can be interpreted as low (about 25\%), moderate (about 50\%) and high (about 75\%) (28).

To determine whether publication bias is present, funnel plot asymmetry was assessed visually and by Egger's regression test. Our meta-analysis was performed with SAS 9.4 edition (SAS Institute).

\section{Results}

\section{MONICA/KORA Augsburg case-cohort study}

Baseline demographic, lifestyle and clinical characteristics of the study participants who developed T2D during the 
follow-up period (cases) and those who remained free of diabetes (non-cases) are reported in Supplementary Table 1.

Risk of incident T2D in the top quartile of fetuin-A was significantly higher than the risk in the bottom quartile after adjustment for age, sex and survey (HR (95\% CI): 1.68 (1.29-2.18)) (model 1, Table 1). This association was slightly attenuated in the model adjusting for established T2D risk factors (1.45 (1.08-1.96)) (model 2). Further adjustment for dietary habits did not influence the association (model 3). The association was similar in models that were individually adjusted for CRP and adiponectin (models 4 and 5). In model 3, an increment per 1 s.D. of log-transformed fetuin-A was associated with a 15\% higher risk of T2D. Results were similar for models 4 and 5.

After sex-stratification elevated concentrations of fetuin-A were significantly associated with an increased risk of T2D among men and women in the models adjusting for age and survey. The HRs were 1.78 (1.242.57) in males and $1.57(1.09-2.28)$ in females for the fourth quartile compared with the first quartile of fetuin-A (Table 1 model 1). Adjustment for established diabetes risk factors (model 2) hardly influenced the HRs in both sexes and the association between fetuin- $\mathrm{A}$ and incident T2D became statistically non-significant (HR females 1.30 (0.83-2.05)). Additional adjustment for dietary habits, CRP and adiponectin influenced the HRs only slightly (models 3-5). Overall, the interaction between fetuin-A and sex was not significant ( $P$ for interaction in model $3=0.62$ ), suggesting that the association between fetuin- $A$ and T2D is similar in males and females.

\section{Systematic meta-analysis}

We identified 7 eligible published articles reporting the association of fetuin-A and incident T2D and included data from the present analysis of the MONICA/KORA casecohort study in the meta-analysis (Supplementary Fig. 1). Studies reporting only separate estimates for males and females $(8,9)$ were considered as different studies when pooling the estimates. Supplementary Table 2 summarizes the characteristics of eligible published articles. The results of quality assessment and rescaling are reported in Supplementary Tables 3 and 4, respectively.

Our meta-analysis included a total of 3106 cases of T2D during a mean/median follow-up time of 2.7-14.3 years. Each S.D. increment in fetuin-A was associated with a $24 \%$ and $28 \%$ higher risk of T2D $(P<0.001)$ using multivariable (Fig. 1) and minimally (Supplementary Fig. 2) adjusted estimates, respectively. There was moderate evidence of heterogeneity when combining study estimates from multivariable adjusted models $\left(P \_Q=0.025 ; \mathrm{I}-\mathrm{sq}=52.7 \%\right)$. Visual inspection of the funnel plot showed that studies with high and low precision of the estimates were distributed symmetrically around the pooled estimate

Table 1 Results from MONICA/KORA Augsburg case-cohort study. Data are presented as hazard ratios (95\% confidence intervals).

\begin{tabular}{|c|c|c|c|c|c|}
\hline \multirow[b]{2}{*}{ Model } & \multicolumn{4}{|c|}{ Fetuin-A quartiles } & \multirow[b]{2}{*}{ Continuous (per 1 s.D.) } \\
\hline & $\mathrm{I}$ & II & III & IV & \\
\hline \multicolumn{6}{|c|}{ All $(n=2704)$} \\
\hline Model 1 & (ref.) & $1.20(0.92-1.57)$ & 1.37 (1.04-1.78) & $1.68(1.29-2.18)$ & 1.19 (1.09-1.30) \\
\hline Model 2 & (ref.) & $1.09(0.81-1.47)$ & $1.27(0.93-1.72)$ & 1.45 (1.08-1.96) & 1.15 (1.04-1.28) \\
\hline Model 3 & (ref.) & $1.09(0.81-1.47)$ & $1.27(0.94-1.73)$ & 1.45 (1.08-1.95) & $1.15(1.04-1.28)$ \\
\hline Model 4 & (ref.) & $1.10(0.81-1.49)$ & $1.28(0.94-1.74)$ & 1.47 (1.09-1.98) & $1.16(1.04-1.28)$ \\
\hline Model 5 & (ref.) & $1.05(0.77-1.44)$ & $1.24(0.91-1.69)$ & 1.47 (1.08-1.99) & $1.17(1.05-1.30)$ \\
\hline \multicolumn{6}{|c|}{ Females $(n=1241)$} \\
\hline Model 1 & (ref.) & $1.06(0.73-1.55)$ & $1.30(0.89-1.90)$ & 1.57 (1.09-2.28) & $1.23(1.08-1.40)$ \\
\hline Model 2 & (ref.) & $0.94(0.59-1.50)$ & $1.17(0.74-1.86)$ & $1.30(0.83-2.05)$ & $1.16(0.99-1.36)$ \\
\hline Model 3 & (ref.) & $0.95(0.60-1.51)$ & $1.17(0.74-1.87)$ & $1.31(0.84-2.07)$ & $1.16(0.99-1.36)$ \\
\hline Model 4 & (ref.) & $0.97(0.61-1.54)$ & $1.18(0.73-1.89)$ & $1.33(0.84-2.09)$ & $1.16(0.99-1.37)$ \\
\hline Model 5 & (ref.) & $0.90(0.55-1.47)$ & $1.17(0.73-1.89)$ & 1.35 (0.85-2.13) & 1.19 (1.01-1.40) \\
\hline \multicolumn{6}{|c|}{ Males $(n=1463)$} \\
\hline Model 1 & (ref.) & $1.31(0.91-1.90)$ & $1.39(0.95-2.04)$ & 1.78 (1.24-2.57) & $1.17(1.04-1.32)$ \\
\hline Model 2 & (ref.) & $1.30(0.86-1.97)$ & $1.38(0.89-2.15)$ & 1.75 (1.17-2.62) & $1.17(1.03-1.34)$ \\
\hline Model 3 & (ref.) & $1.29(0.85-1.96)$ & $1.39(0.90-2.15)$ & $1.74(1.16-2.61)$ & $1.17(1.03-1.34)$ \\
\hline Model 4 & (ref.) & $1.30(0.86-1.98)$ & $1.40(0.90-2.17)$ & 1.75 (1.16-2.63) & $1.18(1.03-1.35)$ \\
\hline Model 5 & (ref.) & $1.25(0.82-1.91)$ & $1.29(0.82-2.03)$ & 1.68 (1.11-2.54) & 1.18 (1.02-1.35) \\
\hline
\end{tabular}


Studies

MONICA/ KORA

Stefan, et al (2008)

Ix, et al (2012)

Aroner, et al (2017)_W

Sun, et al (2013)

Aroner, et al (2017)_M

Ix, et al (2008)

Laughlin, et al (2013)_M

Laughlin, et al (2013)_W

Dutta, et al (2014)

Overall Random Effects

Overall Fixed Effects

Heterogeneity:

P_Q $=0.025 ; \mathrm{l}-\mathrm{sq}=52.7 \%$
N(cases) N(total) wRE

$\begin{array}{rrr}930 & 2704 & 16.1 \% \\ 703 & 2803 & 15.4 \% \\ 305 & 3710 & 14.3 \% \\ 247 & 1173 & 13.0 \% \\ 470 & 940 & 12.6 \% \\ 208 & 974 & 11.4 \% \\ 135 & 518 & 5.7 \% \\ 53 & 477 & 4.7 \% \\ 32 & 782 & 3.6 \% \\ 23 & 120 & 3.3 \% \\ \mathbf{3 1 0 6} & \mathbf{1 4 2 0 1} & . \\ \mathbf{3 1 0 6} & \mathbf{1 4 2 0 1} & . \\ . & . & . \\ . & . & .\end{array}$

Relative Risk (95\% Cl)

RR LCl UCl

$\begin{array}{lll}1.15 & 1.04 & 1.28\end{array}$

$\begin{array}{lll}1.19 & 1.06 & 1.33\end{array}$

$\begin{array}{lll}1.13 & 1.00 & 1.28\end{array}$

$\begin{array}{lll}1.51 & 1.32 & 1.74\end{array}$

$\begin{array}{lll}1.27 & 1.10 & 1.47\end{array}$

$\begin{array}{lll}1.12 & 0.94 & 1.32\end{array}$

$\begin{array}{lll}1.50 & 1.12 & 2.00\end{array}$

$\begin{array}{lll}0.96 & 0.69 & 1.33\end{array}$

$\begin{array}{lll}1.53 & 1.04 & 2.25\end{array}$

$\begin{array}{lll}1.47 & 0.98 & 2.22\end{array}$

$\begin{array}{lll}1.24 & 1.14 & 1.34\end{array}$

$\begin{array}{lll}1.22 & 1.16 & 1.28\end{array}$

\section{Figure 1}

Forest plot summarizing individual study estimates and pooled estimates for the association of a 1 S.D. increment of fetuin-A level with incident T2D adjusted for as many classical T2D risk factors as possible. I-sq, I-squared statistic; $\mathrm{LCl}$ and $\mathrm{UCl}$, lower and upper confidence intervals; $N$ (cases), number of T2D cases; N (total), number of total study participants; $P \_Q, P$ value for Cochran's $Q$ test; RR, relative risk; WRE, random effects weights. $M$ and $W$ indicate sex-specific associations in men and women, respectively. Diamonds represent the overall (pooled) association estimates. Bars indicate $95 \%$ confidence intervals. Dashed vertical line indicates the pooled random effects estimate. The size of the box illustrates the random effects weight. Studies were sorted according to the study's weight in the random effects meta-analysis.

(Supplementary Fig. 3). Thus, no evidence of publication bias was suggested, which was further supported by the non-significant Egger's regression test $(P=0.369)$.

To examine the sex-specific association between fetuin-A and incident T2D, we combined our sexspecific results with 4 previous studies reporting female-specific associations and 3 studies reporting male-specific associations. The pooled multivariable adjusted RRs (95\% CIs) showed a significant positive association in both males and females. The pooled estimates per 1 s.D. increment of fetuin-A in males and females were $1.19(1.04-1.38)$ and 1.29 (1.15-1.46), respectively (Fig. 2).

We further meta-analyzed 5 estimates from 4 studies (1 study reported 2 estimates for males and females separately) investigating the effect of inflammation on the associations. The pooled RRs were 1.26 (1.12-1.41) with and $1.26(1.13-1.40)$ without adjustment for inflammation (Fig. 3).

Three estimates from 3 studies investigating the effect of adiponectin and 6 estimates from 4 studies ( 2 studies reporting 2 estimates for males and females separately) investigating the effect of liver fat content were also combined. The associations remained significant after adjustment for adiponectin and liver fat content separately. The pooled RRs were 1.17 (1.05$1.29)$ and 1.19 (1.01-1.39) with and without adjustment for adiponectin and they were 1.19 (1.08-1.32) and 1.24 (1.10-1.40) with and without adjustment for liver fat content, respectively (Fig. 3). There was no evidence of heterogeneity between studies adjusted for adiponectin and liver fat content.

Subgroup analyses were performed to explore the heterogeneity in our meta-analysis (Supplementary 


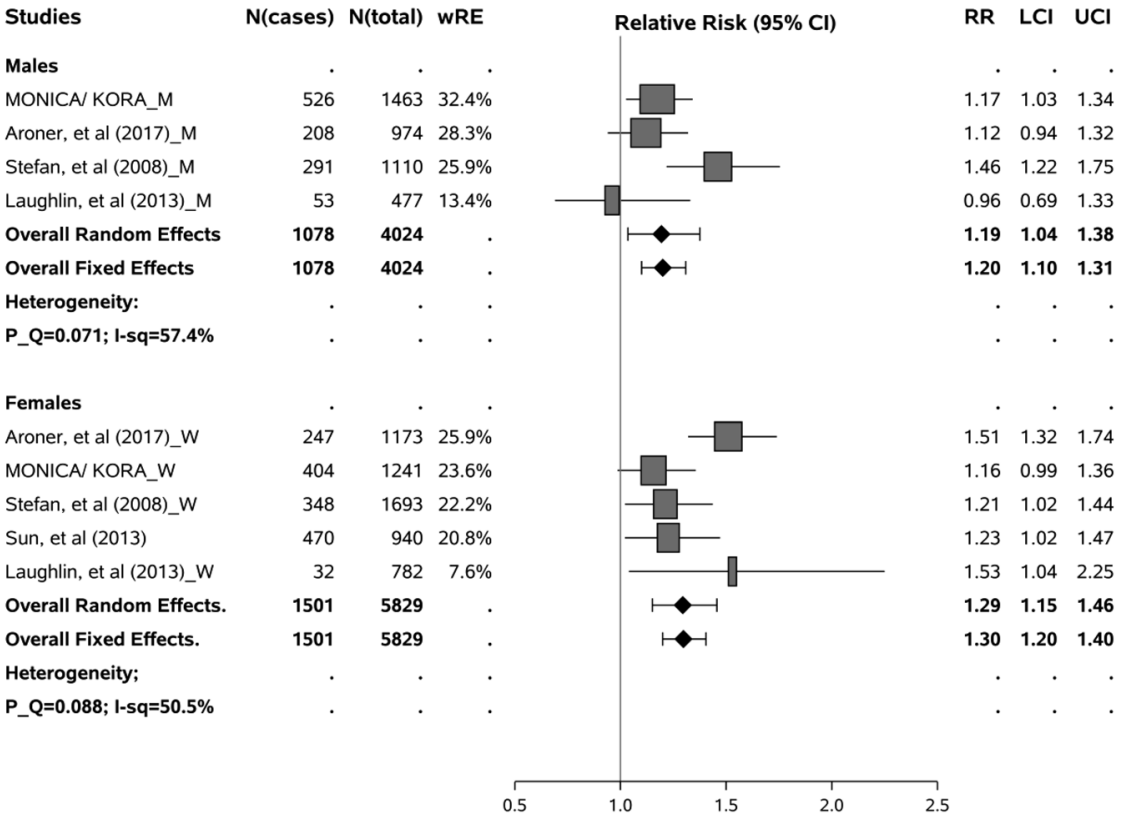

\section{Figure 2}

Forest plot summarizing sex-specific associations per 1 S.D. increment of fetuin-A level with incident T2D adjusted for as many classical risk factors as possible in males and females. I-sq, indicates I-squared statistic; $\mathrm{LCl}$ and $\mathrm{UCl}$, lower and upper confidence intervals; $\mathrm{N}$ (cases) indicates number of T2D cases; $\mathrm{N}$ (total), number of total study participants; $P_{-} Q, P$ value for Cochran's $Q$ test for heterogeneity; RR, relative risk; WRE, random effects weights. $M$ and $W$ indicate sex-specific association in men and women, respectively. Diamonds represent the overall (pooled) association estimates. Bars indicate $95 \%$ confidence intervals. The size of the box illustrates the random effects weight. Studies were sorted according to the study's weight in the random effects meta-analysis.
Fig. 4). Evidence of heterogeneity was no longer significant $\left(P_{-} Q=0.275\right)$ and $\mathrm{I}$-sq was reduced from $52.7 \%$ to $20.3 \%$ after excluding studies that enrolled participants with mixed ethnicities other than Caucasian. The heterogeneity was also reduced by almost $10 \%$ to $43.0 \%$ with $P_{-} Q$ being 0.135 for studies including only older participants ( $\geq 65$ years). We also grouped studies based on the study quality (NOS score) and the number of incident cases comparing those with more versus less than 100 incident cases. However, the results did not explain heterogeneity. Because most of the studies have more than 100 incident cases, we additionally compared those with more vs less cases than the mean number of incident cases. We found no evidence of heterogeneity $\left(P_{-} Q=0.560\right.$; I-sq $\left.=0 \%\right)$ in the 3 largest studies with more than the mean number of incident cases (310 cases).

We conducted sensitivity analyses to examine the robustness of our observations. First, we excluded a study including only pre-diabetic persons to investigate T2D incidence (30). The result did not change substantially: the pooled RR was $1.23(1.13-1.33)$ and $P_{-} Q$ was 0.020 . Second, we excluded the only study which adjusted for IL6 and TNFalpha and not for CRP (10) from our pooled analyses adjusted for inflammation. The pooled RR was 1.23 (1.10-1.38) and the evidence of heterogeneity remained significant $\left(P_{-} Q=0.022\right)$.

\section{Discussion}

\section{Summary of findings}

In this large case-cohort study, which included Caucasian participants from the region of Augsburg, Southern Germany, higher levels of fetuin-A were significantly associated with an elevated risk of incident T2D. The association tended to be slightly weaker among women where associations became non-significant after adjustment for T2D risk factors, but we did not observe any significant sex differences. Adjustment for CRP and adiponectin had only a marginal impact on the strength of observed associations. Our own results from the MONICA/KORA Augsburg case-cohort study are largely in line with the results from the present metaanalysis combining data from 7 published studies and the MONICA/KORA Augsburg case-cohort study. In the meta-analysis, higher fetuin-A levels were significantly associated with risk of incident diabetes among both males and females and adjustment for subclinical inflammation, adiponectin or liver fat content did not explain the observed associations.

\section{Comparison of results with other studies}

The present case-cohort study is the largest individual study addressing the association between fetuin-A and incident 
Model

Adjustment intensity

- Minimum adjustment

- Multivariable adjustment

Inflammation adjustment

- Without inflammation

- With inflammation

Adiponectin adjustment

- Without adiponectin

- With adiponectin

Liver fat content adjustment

- Without liver fat content

- With liver fat content

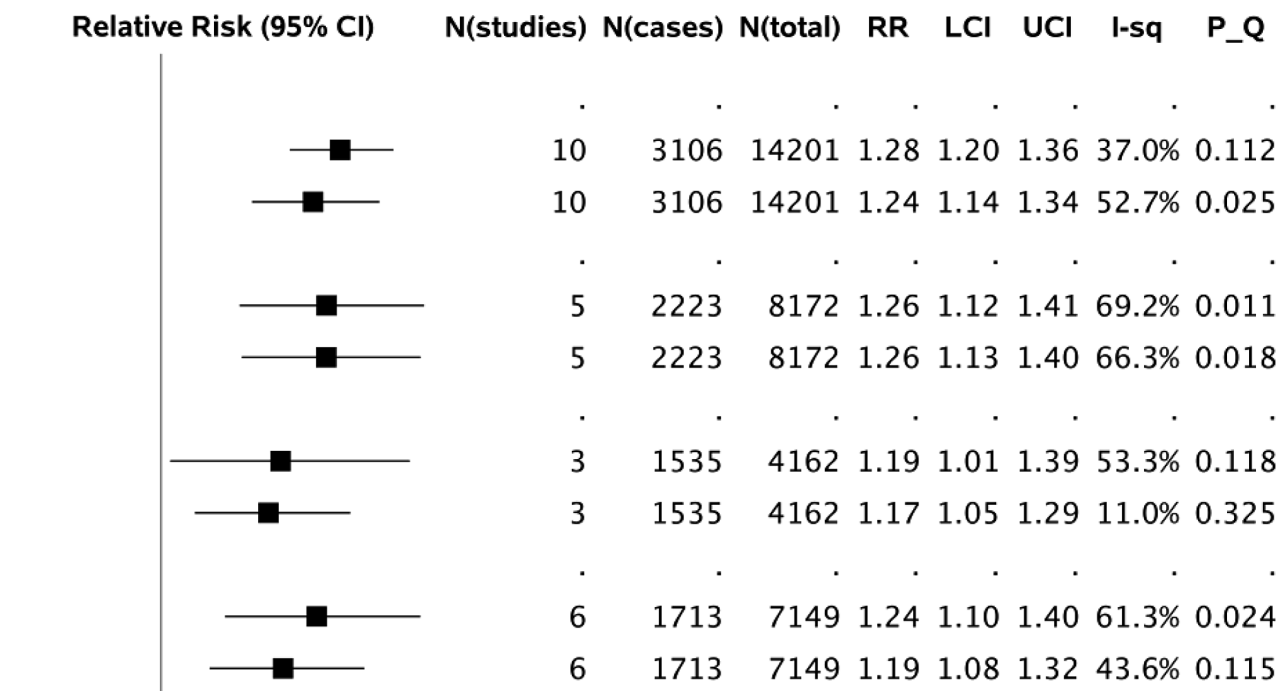

\section{Figure 3}

Forest plot summarizing the results of the main analyses. I-sq indicates I-squared statistic, the proportion of heterogeneity; LCl and $\mathrm{UCl}$, lower and upper confidence intervals; N (cases), number of T2D cases; N(studies) indicates number of studies included; N (total), number of total study participants; $P_{-} Q, P$ value for Cochran's $Q$ test; RR, relative risk. Minimum adjustment indicates model adjusted for age, sex and minimal number of classical risk factors as possible. Multivariable adjustment indicates model adjusted for as many classical risk factors as possible. Bars indicate $95 \%$ confidence intervals.

T2D published to date in terms of included number of incident cases (>900). Thus, the present meta-analysis has a considerably larger sample size (more than 3000 cases of T2D) and consequently a larger power than previous meta-analyses $(8,11,31)$. Furthermore, in extension to the most recent meta-analysis (31), we calculated pooled sex-specific risk estimates to address the unresolved issue of potential sex differences in the association between fetuin-A and incident T2D (8), and we performed extensive further subgroup analyses to identify potential sources of heterogeneity. Furthermore, we specifically addressed the mediating/confounding impact of subclinical inflammation, adiponectin and liver fat content.

Overall, our findings support the results from previous meta-analyses indicating a positive association between fetuin-A and incident T2D $(8,11,31)$. However, while the meta-analysis of Aroner and coworkers suggested that the risk of T2D associated with fetuin-A is higher among women (effect estimate (95\% CI) per 1 s.D. increment: 1.51 (1.32-1.74) (8) than among men (1.12 (0.94-1.32)), we did not observe any significant sex differences.

We found that adjustment for CRP, a biomarker of inflammation and for adiponectin did not affect the association between fetuin-A and incident T2D in the MONICA/KORA Augsburg case-cohort study. Likewise, in our meta-analysis after combining studies, which separately adjusted for inflammation, adiponectin and liver fat content, we found that fetuin-A remained significantly associated with T2D risk. Our meta-analysis is the first to systematically investigate whether inflammation, adiponectin or liver fat content mediate or confound the association between fetuin-A concentrations and T2D risk. Previous individual studies that addressed the impact of subclinical inflammation $(5,8,10)$, adiponectin $(10,11)$ and liver fat content assessed through CT scans (8) or liver enzymes $(5,9,11)$ observed that these factors hardly attenuated the observed associations.

\section{Heterogeneity in study results}

Our meta-analysis revealed moderate evidence of heterogeneity when combining study estimates from multivariable adjusted models $\left(P_{-} Q=0.025\right.$; $\mathrm{I}-\mathrm{sq}=52.7 \%)$. This heterogeneity was not explained by sex. However, further subgroup analysis demonstrated that different ethnic backgrounds and age explain a large 
proportion of the observed heterogeneity. Heterogeneity was reduced to $20.3 \%$ in predominantly Caucasian populations and to $43 \%$ in elderly populations. In the meta-analysis of Roshanzamir et al. (31), heterogeneity was also moderate $\left(P \_Q=0.10 ; \mathrm{I}-\mathrm{sq}=46.1 \%\right.$, but no subgroup analyses were performed due to the limited number of studies.

\section{Mechanisms}

Stefan and Häring suggested that fetuin-A promotes insulin resistance by interacting with free fatty acids (FFAs) (32). Fetuin-A is involved in lipid-induced inflammation by acting as an endogenous ligand between FFAs and Toll-like receptor 4 in adipocytes resulting in insulin resistance $(33,34)$. A recent study also found that in the presence of fatty liver, fetuin-A directly impairs glucose-induced insulin secretion without interacting with Toll-like receptor 4 by mediating metabolic crosstalk between fatty liver and pancreatic islets (35). Furthermore, adiponectin, another protein secreted from adipose tissues, which is also involved in the regulation of insulin sensitivity $(7,36)$, was found to be inversely associated with fetuin-A (37).

Thus, it is plausible that inflammation, decreased adiponectin levels and increased liver fat content mediate or confound the link between fetuin-A and incident T2D. However, this hypothesis is not supported by our results, which demonstrated little change in the effect estimates for fetuin-A after adjustment for the above-mentioned pathways, suggesting that other yet unknown mechanisms explain the association between fetuin- $\mathrm{A}$ and incident T2D. Nonetheless, it should be noted that we cannot totally rule out that imprecision in the measurements is responsible for our results especially with regard to liver fat content. All studies included in our meta-analysis, except Aroner et al. (8), used liver enzymes (alanine transaminase and gamma-glutamyltranspeptidase), a relatively crude measure of liver fat content (38), as surrogate measures of liver fat.

\section{Strengths of the study}

Our study has several strengths. The MONICA/KORA Augsburg case-cohort study has a considerably larger number of incident cases than previous studies. It is population based and uses a prospective design with a long follow-up period. In our meta-analysis, we included different levels of covariate adjustment, investigated sex differences by separately pooling sex-specific estimates and investigated mediation by separately pooling estimates individually adjusted for potential mediators. We tried to explain heterogeneity by subgroup analyses. Furthermore, our meta-analysis strategy to convert the scales of the included estimates into per 1 s.D. increases of fetuin-A, leads to better comparable estimates than previous meta-analyses pooling quantile extremes without rescaling $(11,31)$.

\section{Limitations of the study}

Limitations of this study also warrant consideration. The initial identification of incident cases of diabetes in the MONICA/KORA Augsburg case-cohort study was based on self-report only as no oral glucose tolerance tests have been performed. Although our meta-analysis pooled estimates using different levels of adjustment, residual confounding cannot be ruled out due to the nature of observational studies. The current case-cohort study as well as our metaanalysis identified evidence in predominantly Caucasian populations; thus, we cannot generalize our results to other ethnicities.

\section{Conclusion}

Higher fetuin-A levels are significantly associated with incident T2D in males and females. Our findings based on studies with mainly Caucasian participants suggest that higher fetuin-A levels increase the risk of T2D independently from subclinical inflammation, adiponectin and liver fat content. Further studies are necessary to explore this association among other ethnicities.

\section{Supplementary data}

This is linked to the online version of the paper at https://doi.org/10.1530/ EJE-17-1053.

Declaration of interest

The authors declare that there is no conflict of interest that could be perceived as prejudicing the impartiality of this study.

\section{Funding}

Cornelia Huth is supported by intramural funding for Translational \& Clinical Projects of the Helmholtz Zentrum München-German Research Center for Environmental Health, Germany, which is funded by the BMBF, Germany and the State of Bavaria. Data analyses of the MONICA/ KORA Augsburg case-cohort study were supported by a grant from the Else Kröner-Fresenius-Stiftung. The KORA study was initiated and financed by the Helmholtz Zentrum München-German Research Center for Environmental Health, which is funded by the BMBF and the State of 
Bavaria. Furthermore, KORA research was supported within the Munich Center of Health Sciences (MC-Health), Ludwig-Maximilians-Universität, as part of LMUinnovativ. The German Diabetes Center is supported by the Ministry of Science and Research of the State of North Rhine-Westphalia (MIWF NRW) and the German Federal Ministry of Health (BMG). This study was supported in part by a grant from the German Federal Ministry of Education and Research (BMBF) to the German Center for Diabetes Research (DZD). The sponsors had no role in study design, data collection, data analysis, data interpretation, writing of the report and decision to publish the manuscript.

\section{Author contribution statement}

The protocol for systematic review and meta-analysis was developed by C S, B T and C Hu. C S conducted the literature search. C S and S M independently conducted data extraction and quality assessment. B T, A $\mathrm{P}, \mathrm{C} \mathrm{He}, \mathrm{W} \mathrm{K}$ and $\mathrm{J} \mathrm{S}-\mathrm{K}$ researched data in the MONICA/KORA Augsburg study. A Z performed statistical analyses for the MONICA/KORA Augsburg case-cohort study. C S performed statistical analyses for the meta-analysis and drafted the manuscript. All authors critically revised the manuscript and approved of the final version.

\section{References}

1 Ogurtsova K, da Rocha Fernandes JD, Huang Y, Linnenkamp U, Guariguata L, Cho NH, Cavan D, Shaw JE \& Makaroff LE. IDF diabetes atlas: global estimates for the prevalence of diabetes for 2015 and 2040. Diabetes Research and Clinical Practice 2017128 40-50. (https://doi.org/10.1016/i.diabres.2017.03.024)

2 Roglic G. WHO Global report on diabetes: a summary. International Journal of Noncommunicable Diseases 20161 3-8. (https://doi. org/10.4103/2468-8827.184853)

3 Stumvoll M, Goldstein BJ \& van Haeften TW. Type 2 diabetes: principles of pathogenesis and therapy. Lancet 2005365 1333-1346. (https://doi.org/10.1016/S0140-6736(05)61032-X)

4 Mori K, Emoto M, Yokoyama H, Araki T, Teramura M, Koyama H, Shoji T, Inaba M \& Nishizawa Y. Association of serum fetuin-A with insulin resistance in type 2 diabetic and nondiabetic subjects. Diabetes Care 200629 468. (https://doi.org/10.2337/diacare.29.02.06. dc05-1484)

5 Stefan N, Fritsche A, Weikert C, Boeing H, Joost HG, Haring HU \& Schulze MB. Plasma fetuin-A levels and the risk of type 2 diabetes. Diabetes 200857 2762-2767. (https://doi.org/10.2337/db08-0538)

6 Ix JH, Biggs ML, Mukamal KJ, Kizer JR, Zieman SJ, Siscovick DS, Mozzaffarian D, Jensen MK, Nelson L, Ruderman $\mathrm{N}$ et al. Association of fetuin-A with incident diabetes mellitus in community-living older adults: the cardiovascular health study. Circulation $2012 \mathbf{1 2 5}$ 2316-2322. (https://doi.org/10.1161/ CIRCULATIONAHA.111.072751)

7 Stefan N, Sun Q, Fritsche A, Machann J, Schick F, Gerst F, Jeppesen C, Joost HG, Hu FB, Boeing $\mathrm{H}$ et al. Impact of the adipokine adiponectin and the hepatokine fetuin-A on the development of type 2 diabetes: prospective cohort- and crosssectional phenotyping studies. PLoS ONE 20149 e92238. (https:// doi.org/10.1371/journal.pone.0092238)

8 Aroner SA, Mukamal KJ, St-Jules DE, Budoff MJ, Katz R, Criqui MH, Allison MA, de Boer IH, Siscovick DS, Ix JH et al. Fetuin-A and risk of diabetes independent of liver fat content: the multi-ethnic study of atherosclerosis. American Journal of Epidemiology 2017185 54-64. (https://doi.org/10.1093/aje/kww095)

9 Laughlin GA, Barrett-Connor E, Cummins KM, Daniels LB, Wassel CL \& Ix JH. Sex-specific association of fetuin-a with type 2 diabetes in older community-dwelling adults: the Rancho Bernardo study. Diabetes Care 201336 1994-2000. (https://doi.org/10.2337/ dc12-1870)
10 Ix JH, Wassel CL, Kanaya AM, Vittinghoff E, Johnson KC, Koster A, Cauley JA, Harris TB, Cummings SR \& Shlipak MG. Fetuin-A and incident diabetes mellitus in older persons. JAMA 2008300 182-188. (https://doi.org/10.1001/jama.300.2.182)

11 Sun Q, Cornelis MC, Manson JE \& Hu FB. Plasma levels of fetuin-A and hepatic enzymes and risk of type 2 diabetes in women in the U.S. Diabetes 201362 49-55. (https://doi.org/10.2337/db12-0372)

12 Herder C, de Las Heras Gala T, Carstensen-Kirberg M, Huth C, Zierer A, Wahl S, Sudduth-Klinger J, Kuulasmaa K, Peretz D, Ligthart S et al. Circulating levels of interleukin 1-receptor antagonist and risk of cardiovascular disease: meta-analysis of six populationbased cohorts. Arteriosclerosis, Thrombosis, and Vascular Biology 201737 1222-1227. (https://doi.org/10.1161/ATVBAHA.117.309307)

13 Thorand B, Kolb H, Baumert J, Koenig W, Chambless L, Meisinger C, Illig T, Martin S \& Herder C. Elevated levels of interleukin-18 predict the development of type 2 diabetes: results from the MONICA/KORA Augsburg Study, 1984-2002. Diabetes 200554 2932-2938. (https:// doi.org/10.2337/diabetes.54.10.2932)

14 Meisinger C, Thorand B, Schneider A, Stieber J, Döring A \& Löwel H. Sex differences in risk factors for incident type 2 diabetes mellitus: the monica augsburg cohort study. Archives of Internal Medicine 2002162 82-89. (https://doi.org/10.1001/ archinte.162.1.82)

15 Thorand B, Baumert J, Kolb H, Meisinger C, Chambless L, Koenig W $\&$ Herder C. Sex differences in the prediction of type 2 diabetes by inflammatory markers: results from the MONICA/KORA Augsburg case-cohort study, 1984-2002. Diabetes Care 200730 854-860. (https://doi.org/10.2337/dc06-1693)

16 Thorand B, Zierer A, Baumert J, Meisinger C, Herder C \& Koenig W. Associations between leptin and the leptin/adiponectin ratio and incident Type 2 diabetes in middle-aged men and women: results from the MONICA/KORA Augsburg study 1984-2002. Diabetic Medicine 201027 1004-1011. (https://doi.org/10.1111/j.14645491.2010.03043.x)

17 Wilsgaard T, Mathiesen EB, Patwardhan A, Rowe MW, Schirmer H, Lochen ML, Sudduth-Klinger J, Hamren S, Bonaa KH \& Njolstad I. Clinically significant novel biomarkers for prediction of first ever myocardial infarction: the Tromso Study. Circulation: Cardiovascular Genetics 20158 363-371. (https://doi.org/10.1161/ CIRCGENETICS.113.000630)

18 Barlow WE. Robust variance estimation for the case-cohort design. Biometrics 199450 1064-1072. (https://doi.org/ $10.2307 / 2533444)$

19 van Buuren S, Boshuizen HC \& Knook DL. Multiple imputation of missing blood pressure covariates in survival analysis. Statistics in Medicine 199918 681-694. (https://doi.org/10.1002/(SICI)10970258(19990330)18:6<681::AID-SIM71>3.0.CO;2-R)

20 R Core Team. R: A Language and Environment for Statistical Computing. Vienna, Austria: R Foundation for Statistical Computing, 2015.

21 van Buuren S \& Groothuis-Oudshoorn K. mice: multivariate imputation by chained equations in R. Journal of Statistical Software 20114567.

22 Rubin DB. Multiple Imputation for Nonresponse in Surveys. New York, NY: J. Wiley \& Sons, 1987.

23 DerSimonian R \& Laird N. Meta-analysis in clinical trials. Controlled Clinical Trials 19867 177-188. (https://doi.org/10.1016/01972456(86)90046-2)

24 DerSimonian R \& Kacker R. Random-effects model for meta-analysis of clinical trials: an update. Controlled Clinical Trials 200728 105-114. (https://doi.org/10.1016/j.cct.2006.04.004)

25 Normand S-LT. Meta-analysis: formulating, evaluating, combining, and reporting. Statistics in Medicine 199918 321-359. (https:// doi.org/10.1002/(SICI)1097-0258(19990215)18:3<321::AIDSIM28>3.0.CO;2-P)

26 Orban E, Schwab S, Thorand B \& Huth C. Association of iron indices and type 2 diabetes: a meta-analysis of observational studies. 
Diabetes/Metabolism Research and Reviews 201430 372-394. (https:// doi.org/10.1002/dmrr.2506)

27 Cochran WG. The combination of estimates from different experiments. Biometrics 195410 101-129. (https://doi. org $/ 10.2307 / 3001666$ )

28 Higgins JPT, Thompson SG, Deeks JJ \& Altman DG. Measuring inconsistency in meta-analyses. BMJ 2003327 557-560. (https://doi. org/10.1136/bmj.327.7414.557)

29 Higgins JP \& Thompson SG. Quantifying heterogeneity in a metaanalysis. Statistics in Medicine 200221 1539-1558. (https://doi. org/10.1002/sim.1186)

30 Dutta D, Mondal SA, Kumar M, Hasanoor Reza AH, Biswas D, Singh P, Chakrabarti S \& Mukhopadhyay S. Serum fetuin-A concentration predicts glycaemic outcomes in people with prediabetes: a prospective study from eastern India. Diabetic Medicine 201431 1594-1599. (https://doi.org/10.1111/ dme.12539)

31 Roshanzamir F, Miraghajani M, Rouhani MH, Mansourian M, Ghiasvand R \& Safavi SM. The association between circulating fetuin-A levels and type 2 diabetes mellitus risk: systematic review and meta-analysis of observational studies. Journal of Endocrinological Investigation 201741 33-47. (https://doi.org/10.1007/s40618-0170697-8)
32 Stefan N \& Häring HU. Circulating fetuin-A and free fatty acids interact to predict insulin resistance in humans. Nature Medicine 2013 19 394-395. (https://doi.org/10.1038/nm.3116)

33 Heinrichsdorff J \& Olefsky JM. Fetuin-A: the missing link in lipidinduced inflammation. Nature Medicine 201218 1182-1183. (https:// doi.org/10.1038/nm.2869)

34 Pal D, Dasgupta S, Kundu R, Maitra S, Das G, Mukhopadhyay S, Ray S, Majumdar SS \& Bhattacharya S. Fetuin-A acts as an endogenous ligand of TLR4 to promote lipid-induced insulin resistance. Nature Medicine 201218 1279-1285. (https://doi.org/10.1038/nm.2851)

35 Gerst F, Wagner R, Kaiser G, Panse M, Heni M, Machann J, Bongers MN, Sartorius T, Sipos B, Fend F et al. Metabolic crosstalk between fatty pancreas and fatty liver: effects on local inflammation and insulin secretion. Diabetologia 2017.

36 Ye R \& Scherer PE. Adiponectin, driver or passenger on the road to insulin sensitivity? Molecular Metabolism 20132 133-141. (https:// doi.org/10.1016/j.molmet.2013.04.001)

37 Hennige AM, Staiger H, Wicke C, Machicao F, Fritsche A, Haring HU $\&$ Stefan N. Fetuin-A induces cytokine expression and suppresses adiponectin production. PLOS ONE $20083 \mathrm{e} 1765$. (https://doi. org/10.1371/journal.pone.0001765)

38 Rinella ME. Nonalcoholic fatty liver disease: a systematic review. JAMA 2015313 2263-2273. (https://doi.org/10.1001/jama.2015.5370) 